DOI: 10.20472/IAC.2018.042.039

\author{
ANDREA POTGIETER \\ University of Johannesburg , South Africa \\ CHRIS RENSLEIGH \\ University of Johannesburg, South Africa
}

\title{
DESIGNING FOR USER EXPERIENCE: ANALYSING APP STORE REVIEWS FOR APP FEATURE IDENTIFICATION
}

\begin{abstract}
:
South Africa's blood stock level is often categorised as alarmingly low, leaving blood donation organisations in constant need of voluntary, unpaid blood donations to ensure their ability to supply hospitals with safe blood. Globally, there are successful mobile blood donation apps facilitating blood donation by providing useful services to blood donors, however similar apps available in South Africa are new, and not popular when compared to global standards. An estimated 5.9 million South Africans download and use mobile applications (apps), and this paper explores the process and results from the first phase of a study, which employed a sequential mixed method research design, to identify user-preferred features for a mobile blood donation app. The findings of the study should serve as a roadmap to blood donation organisations in South Africa, regarding what users expect from a blood donation app, and which features may possibly stimulate a constant or increased frequency of blood donation instances. The two largest app stores, Google Play and Apple iOS, served as the sources of the eventual sample of blood donation apps, of which the user reviews were analysed. Commenting from a design science paradigm, this paper reports on the selection process that had been followed to sample the relevant apps, and further discusses the user insights gained from the analysis of these apps' reviews. The paper further reports on how the app review analysis findings informed the creation of an interview schedule, that was used to gain in-depth understanding of perceptions held by users of the blood donation apps, specifically regarding the users' preferred features in these types of apps.
\end{abstract}

\section{Keywords:}

Mobile application features; Mobile blood donation applications; Design science research; Design thinking, App store reviews

JEL Classification: L31, L86, 033 


\section{Introduction}

Despite constant education drives to increase blood donation numbers, the biggest Blood Donation Organisation (BDO) in South Africa "...still never [has] enough people who are consistently donating" (Eyewitness News, 2016). This BDO operates in eight of South Africa's nine provinces, and the blood is distributed nationally as needed (John, 2016). South Africa is one of 65 countries that rely on voluntary, unpaid blood donations, a practice that is supported by the World Health Organisation (WHO) (Eyewitness News, 2016). According to the WHO (Eyewitness News, 2016), voluntary blood donors who donate blood regularly are the foundation of an adequate national blood supply, as these individuals are associated with lower blood infection that can be transmitted through transfusions.

A blood shortage in South Africa would consequently result in an inability to supply hospitals with safe blood (John, 2016), placing patients in dire need of a blood transfusion, in a life threatening position. A representative of the relevant BDO noted in June 2016 that, unless South Africans get actively involved in the quest to build a sustainable blood stock level, this situation will not improve (Fisher, 2016). Considering that an estimated 5.9 million South Africans download mobile apps (Mobile Marketing Association South Africa (MMAS), 2015:18), the proposed research will aim at identifying mobile app features that could potentially motivate individuals to become actively involved in the blood drive, resulting in such an app acting as a catalyst towards increased blood donation.

\section{An app for social good}

As smartphone adoption increases globally, the popularity of mobile applications (apps) is also increasing radically (Ouhbi, Fernández-Alemán, Pozo, El Bajta, Toval \& Idri, 2015:62). Since low-income countries have an average blood donation rate nine times lower than high-income countries (Eyewitness News, 2016), Ouhbi, Fernández-Alemán, Toval, Idri and Pozo (2015:16) highlight the importance of blood donation apps being specifically developed for the African landscape, as these apps are serving a "noble cause". This sentiment is echoed by the WHO Director-General, Dr Margaret Chan, who said: "Voluntary unpaid blood donation is the act of giving life - the greatest gift any person can give or receive" (Eyewitness News, 2016).

According to Pule, Rachaba, Magafu and Habte (2014:5), simply being adequately informed about blood transfusion and its ability to save lives, can make individuals more likely to intend to donate blood. This implies that, should a mobile app be used only to fulfil the rudimentary function of sharing vital information about blood transfusion and its impact, individuals could already be enticed to be more likely to intend to donate blood. However, the opportunities for user interaction through mobile apps is vast (Kim, Wang \& Malthouse, 2015:37) and information sharing is simply one of many potential features of mobile apps (ITBusiness, 2014; Khandavalli, 2014; Kumar, 2015). 
In a study focusing on apps aimed at crowdsourcing for community activism, Massung, Coyle, Cater, Jay and Preist (2013:376) note app functionality as a considerable impact factor critical to users' engagement in online interaction. These authors go into detail regarding which features of different apps were found appealing by users. For example, collecting badges for the completion of tasks did not have a significant effect on users' behaviour, while an intuitive app which was easy to use seemed to be an important consideration for user satisfaction and therefore interaction (Massung et. al., 2013:376). Similarly, it is the aim of this paper to determine, by analysing mobile blood donation app reviews, which app features blood donors and potential blood donors prefer and which of features garnered the most feedback, be it positive or negative.

\section{Research design \& methodology}

To answer the established research question, a researcher should present a general plan of action, illustrating how these research questions will be answered (Saunders, Lewis \& Thornhill, 2012:159). The following discussion will therefore propose the paper's theoretical and philosophical foundations, as well as a "...framework for the collection and analysis of data" (Bryman \& Bell, 2015:28). The ensuing elements will provide context and a plan of execution for the proposed study.

\subsection{Philosophical Paradigm}

Pragmatism formed the philosophical position during this study. Pragmatism values the practical consequences of research findings (Saunders, et.al., 2012:130) and since the aim of this paper was to provide insight into feedback from mobile blood donation app users, the functional significance of the research findings may be considerable. As noted by Gray (2014:28), pragmatism is also regarded as valuable for "...the insights it has provided for research into management and organisations...", further solidifying its aptness in providing actionable feedback to any stakeholders that could benefit from an effective mobile blood donation app.

\subsection{Research Paradigm \& Methodological Choice}

The research paradigm for this paper, was a qualitative, mono method. However, it must be mentioned that this paper forms part of an ongoing $\mathrm{PhD}$ study, which employs an exploratory sequential mixed methods design. As such, the researcher used qualitative data and analysis during a first phase of enquiry (the data on which this paper is based), and continued with a second, quantitative phase based on the findings revealed in the first phase (Creswell, 2014:225; Saunders, et. al., 2012:167).

The philosophical position of the overall study supports the use of a mixed methods design, as the pragmatist approach "...views the mixing of quantitative and qualitative data in a single study not only as legitimate, but in some cases, necessary" (Gray, 2014:29). In this specific case, it is argued that the use of a mixed method design was in fact necessary, as 
the qualitative component facilitated the quantitative component by developing premises that could be tested during the quantitative component of the larger study. Secondly, the qualitative component aided overall measurement by informing the development of a quantitative data collection tool, through drawing on the in-depth knowledge gained through the initial qualitative approach (Bryman \& Bell, 2014:63).

\subsection{Research Approach}

Abductive logic, the research approach adopted for this study, is based on the pragmatist perspective and seeks to identify conditions that would make a complex issue "less puzzling" (Bryman \& Bell, 2015:9). According to Saunders et. al. (2012:147), abduction combines deduction and induction, moving back and forth between theory and data, which speaks to the daily practices of management and business researchers.

Dunne and Dougherty (2016:154) suggest that innovation in product development, such as the conceptualisation of a mobile app, might benefit from "...integrating the role of abductive reasoning...", as an abductive strategy aids in managing complexity. In a product design context (such as the conceptualisation of an app), abductive reasoning not only aids in countering negative deductive logic, it also allows innovators of the product to employ "design thinking" (Dong, Lovallo \& Mounarath, 2015:55).

Finally, abductive reasoning was beneficial to this study, as it is also seen as a strategy for the researcher to remain open to new interpretations of the data, "...rather than using it to confirm their preunderstandings" (Bryman \& Bell, 2015:9). This implies that abduction acknowledges the researcher's ability to "...think rationally in terms of computational reasoning..." (Bryman \& Bell, 2015:9). Therefore, the researcher's personal perceptions about the context of the study or the expected outcome of the data become less relevant, as conclusions are framed by not only data or theory, but an interaction of those elements.

\subsection{Data Collection Technique(s)}

To identify general themes relevant to users' experiences with existing blood donation apps, an extant collection of customer reviews of current blood donation apps was conducted. This category of data collection focuses on collecting text that was created "...independently of any intervention, influence or prompts by the researcher" (Sloan \& Quan-Haase, 2017:182). Given the voluntary nature of app store reviews, these evaluations often provide user-generated suggestions for improvement of an app, as well as requests for certain app features (lacob \& Harrison, 2013:41).

A collection of app store reviews was used to consider users' experiences of popular blood donation apps available for download on the two largest online app stores, Google Play and the Apple iOS stores (Statista, 2016). The Google Play store allows users to download apps that are compatible with the Android operating system, while the Apple iOS store serves Apple iPhone clients with downloadable apps specifically designed for the iOS hardware. It is important to note that this content analysis mainly looked to international 
apps as currently, there are only two blood donation apps specifically serving South Africa. The first app, developed by the country's largest blood donation organisation, is a simple platform that challenges users to "... a simple pledge [every day] for a year..." and employs the hashtag \#calendarforgood. The aim here is simply to "...help start a revolution..." for South Africans to make a difference to those around them (React Technology, 2016). However, this app does not aim at engaging with existing or potential blood donors to stimulate increased blood donation or awareness. To date, the app has only received one review.

Another app, developed by a smaller blood donation organisation in South Africa, was relevant to the focus of this study in terms of its user-focused features, but was only launched in July of 2017. Therefore, at the time of data collection, this app had only garnered a combined 27 reviews on the two app stores in question. Since this app offered many of the features of other, more popular, blood donation apps, it was included in the qualitative analysis focussing on app store reviews.

\subsection{Sampling App Store Reviews}

As shown in Figure 1, the Google Play and Apple App stores are the leaders in terms of number of apps available for download (Statista, 2017). Furthermore, Figure 2 indicates that the global market share for mobile operating systems (OS), is dominated by Android phones, with Apple iOS following second, although with a considerably lower percentage of the market, compared to Android (Statista, 2017a). Considering the popularity of these two OS, the Google Play and Apple iOS stores were selected as sources of the sample of blood donation apps' app store reviews.

The international Google Play store can be searched with relevant ease, giving Android users access to any apps shown or returned via a search query. However, when searched directly, the Apple iOS store takes into consideration the location of the user, influencing the search results by returning apps that are only available in the user's location. Because of this limitation, a website called fnd.io was used to conduct the search of the Apple iOS store. In fnd.io, the American Apple iOS store is the default search location, but a search done on this website returns apps from international location as well, as is evident from the app 'Organ Donation New Zealand' being part of the initial search results (see Figure 3).

Hennink, Hutter and Bailey (2011:85) are clear in reminding researchers that, even though qualitative research methods employ purposive sampling techniques, the selection of a sample should be done in a methodical and ordered manner. The selection process for the app sample, of which all recorded reviews were analysed, is illustrated in Figure 3. 
Figure 1: Number of apps available in leading app stores as of March 2017 (Statista, 2017)

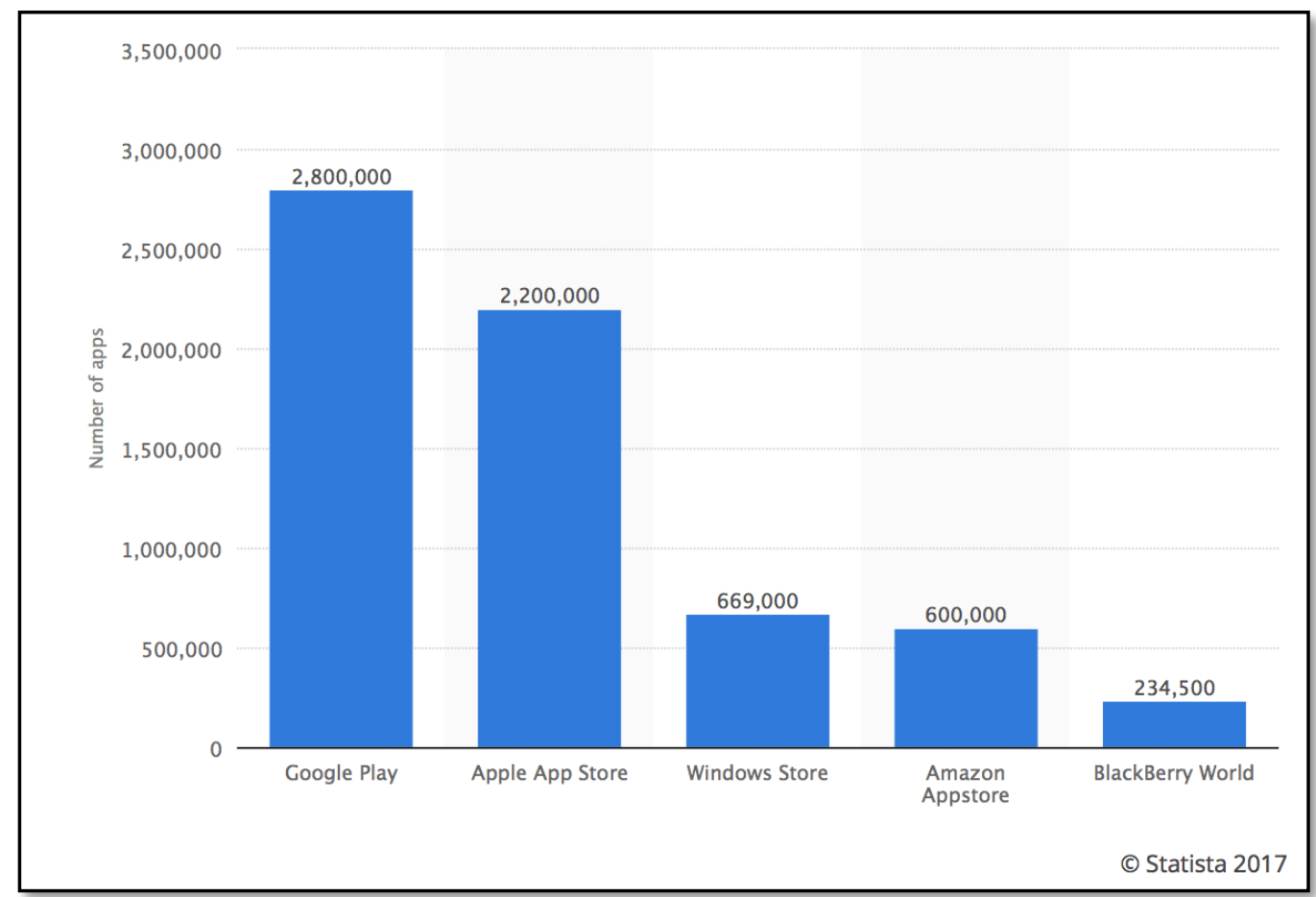

Figure 2: Global mobile OS market share in sales to end users from 1st quarter 2009 to 1 st quarter 2017 (Statista, 2017a)

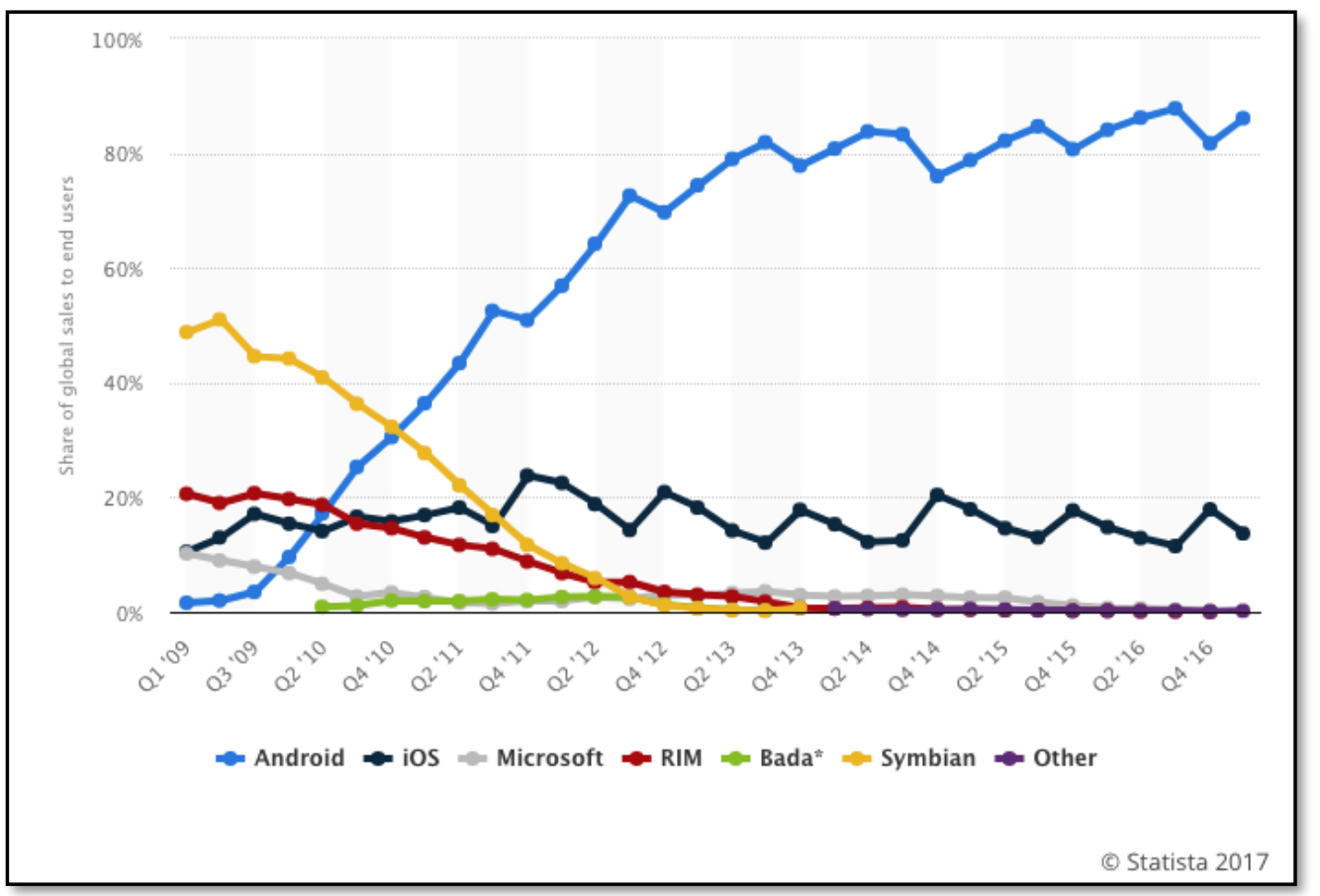


Figure 3: Process explained - App selection for extraction of user reviews

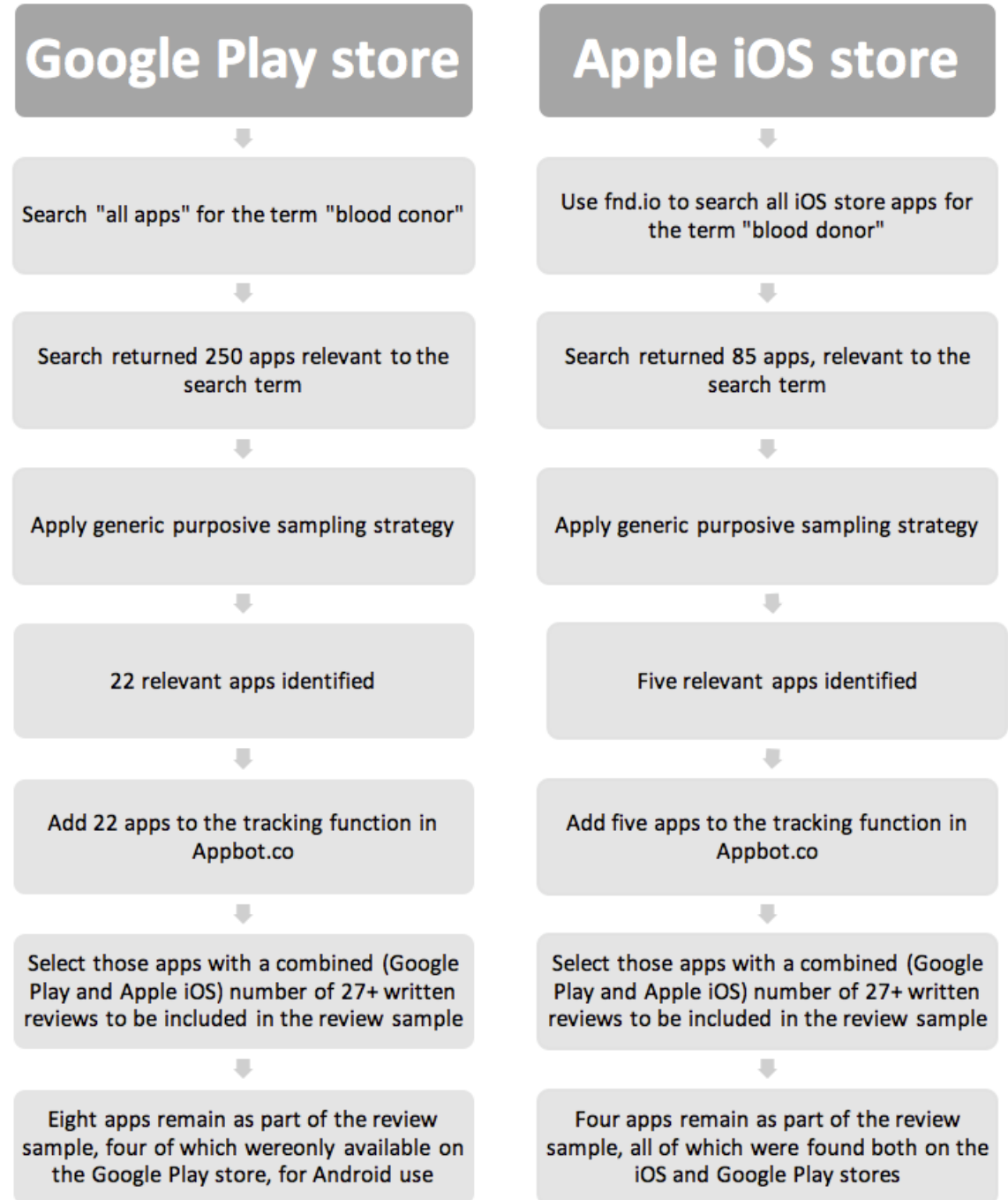

The sampling of apps from which to extract user reviews, was conducted during the week of $16-20$ October 2017. A search for the term "blood donor", within all the available apps in both relevant app stores, was conducted. In the Google Play store, this search action returned 250 relevant apps, while the search in the Apple iOS store delivered 85 relevant apps. Although the searches in both app stores delivered a significant number of apps, it must be noted that many of these apps were not related to blood donation or blood donor support. For example, in Figure 4, several examples of apps returned from each app store are shown.

Apart from the obvious non-relevant apps being excluded during the sampling process, the remaining apps were also scrutinised through employing generic purposive sampling. As described by Bryman and Bell (2015:323), generic purposive sampling allows the sampling to be fixed and a priori. The fixed, a priori criteria that was applied to the sampling of the apps, was informed by the study's research questions (Bryman \& Bell, 2015:323) and 
justification resting on rational intuitions and insights (Russel, 2014). Apps that were included in this phase of the sampling process, adhered to the following criteria:

- The app had to be free;

- The app description had to be in English;

- The app had to have received a star rating by at least 100 individuals; and

- The deducible purpose of the app had to be focused on at least one of the following conditions:

$\checkmark$ Improvement of the blood donor experience,

$\checkmark$ A goal of increased interaction with blood donors, to raise awareness around blood donation, and

$\checkmark$ To attract blood donors to increase the frequency of their blood donations.

Hsu, Lin, Fu and Hung (2015:54) state that app users are typically motivated to pay for an app if they perceive such an app as "...offering value-for-money." Therefore, offering an app for free is a "...very powerful motivator..." to entice users to download the app (Wooldridge \& Schneider, 2010:107). Furthermore, World Health Organisation (WHO) has set a goal for countries worldwide to base their transfusion services on non-remunerated blood donation by 2020 , since "...[v]oluntary, non-remunerated blood donation has been universally shown to be the cornerstone of safe blood" (Asif \& Hassan, 2015:1). Considering the motivational aspect of providing a free app, and taking into consideration that blood donation typically relies on voluntary action, it stands to reason that an app focussing on increasing user interaction and increased awareness of blood donation, should be free. Finally, all the blood donation-specific apps identified during this phase of sampling were, in fact, free.

Only apps of which the app store description was done in English, were included in the sample. The reason for this selection criteria, was purely practical as Leximancer, the data analysis tool used to analyse the sample, was limited in which languages it can process automatically (Leximancer, 2013).

Furthermore, only those apps that had garnered more than 100 star ratings on the relevant app stores, were considered for the sample. App users can rate a downloaded app using a scale of one to five stars. Users can also write a review for a downloaded app; reviews are a subset of app ratings, since "...a review is not required to rate an app, but a rating is required to review an app" (Ruiz, Nagappan, Adams, Berger, Dienst \& Hassan, 2017:1). PowerReviews (2014:34) reports that $95 \%$ of consumers consult customer reviews before making a purchase, indicating their value in terms of customers' decision whether or not to download an app. Star ratings also impact the discoverability of an app on an app store (Sefferman, 2016), suggesting that the more ratings an app has received, the more times 
it has been downloaded, and the more opportunity there has been for a user to write a review for the app - the data that this sampling method had as end-goal.

As mentioned, the remaining criteria for selecting apps for the sample, was linked to the study's research questions. The researcher evaluated each description of those apps that met the initial criteria, and purposively selected only those apps that met at least one of the conditions linked to the study's research questions.

Figure 4: Examples of apps returned from search, but unrelated to blood donation

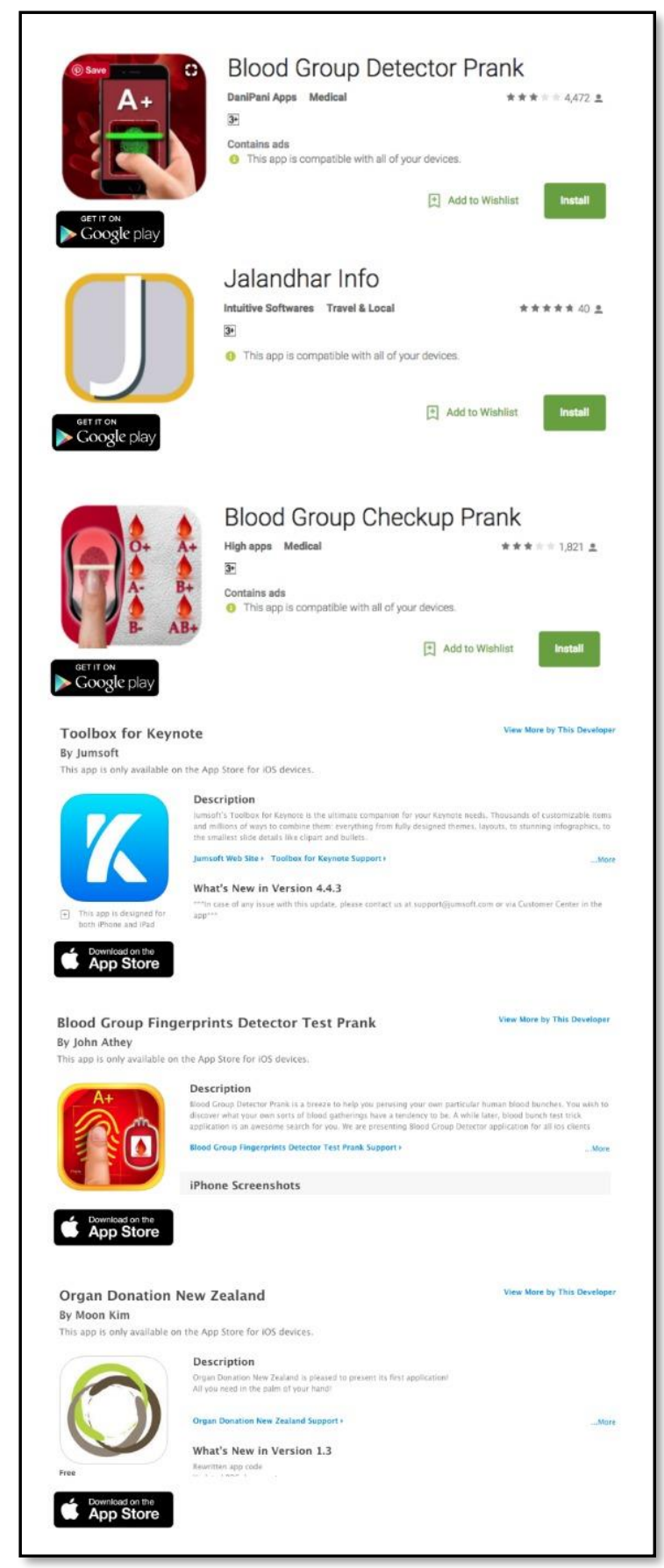


After this point in the sampling process, the researcher had selected a preliminary sample of 22 apps. Of those 22 apps, five were found on both the Google Play store and the Apple iOS store, with the remaining 17 apps only being available to Android users on the Google Play store. These 22 apps were added to Appbot.co, a software start-up that assists app developers in "understand[ing] what their customers want by analysing app reviews" (AngelList, n.d). Appbot.co functions by clustering reviews by various categories to identify customer needs and product improvement opportunities (AngelList, n.d). This platform served as a practical way to manage and track all the apps this study would be regarding for reviews, in one place. The Appbot.co "Compare Apps" function also allowed the researcher to further study the initial sample, as the number of reviews that each app had received since 9 July 2008, was displayed in the dashboard of this function.

Again, purposive sampling was applied, however in this instance the researcher only regarded the number of reviews an app had received since its launch. If an app acquired 27 or more reviews, it was included in the final sample. In the cases of apps that were available on both the Google Play store and Apple iOS store, the total number of reviews from both stores was considered. The reason for this selection number, was that a blood donation app by one of the two blood donation organisations in South Africa, was purposefully selected for inclusion into the final sample, although it had not received more than 100 star ratings.

Though this app had only received 41 star ratings and 26 reviews on the Google Play store, and one review and rating on the Apple iOS store, the data of the app's reviews was considered strategically relevant in answering the study's research questions, as it was the only South African-specific app (Bryman \& Bell, 2015:319). Since this app had only received 27 reviews in total, it was decided that 27 would be the lowest number of reviews that would be considered for any app selected in the previous phase of sampling, to be included in the final sample. According to Johannesson and Perjons (2014:65), a weakness of content analysis, is its insensitivity to context, as individual units are analysed; in the case of this research, those units were user reviews. By including the only available user reviews from South Africans using a blood donation app which met the study's research question requirements, the researcher attempted to incorporate context into the data that was analysed, even though the relevant app's reviews only accounted for $0.7 \%$ of the total number of apps reviewed.

This final phase of sampling produced a sample of twelve apps, four of which were represented on both the Google Play and the Apple iOS store. These twelve apps are shown in Figure 5, arranged by number of reviews in a descending order. The total number of reviews that would be downloaded through Appbot.co, amounted to 3699 reviews. 
Figure 5: Final sample of Google Play and Apple iOS store apps

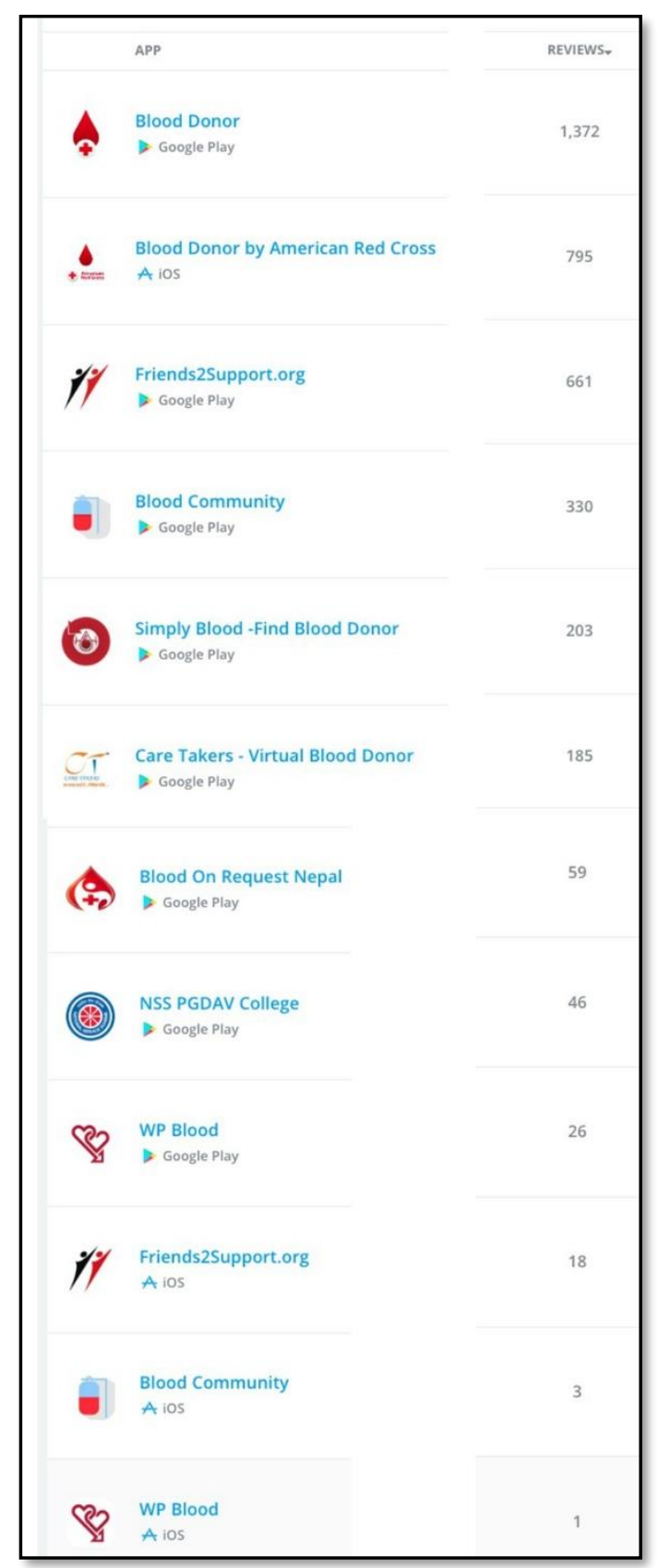

\subsection{Analysis of App Store Reviews}

An analysis of app store reviews focused on identifying themes that arose from the content being analysed, with the potential for "...refinement of those categories and the generation of new ones" (Bryman \& Bell, 2015:404). The aim of the content analysis of the sampled apps' app store reviews, was to inform the focus of the asynchronous online interviews, which were conducted after the conclusion of the app store review analysis. The app store analysis, in turn, was interpreted in comparison to existing literature regarding users' 
preferences and motivations regarding mobile app features. By employing triangulation in such a manner, the researcher could better understand the 'how' and 'why' regarding preferred features of mobile app users (Salmons, 2016:123).

An Appbot.co subscription allowed the researcher to download all the existing reviews for the sampled apps, in 12 separate .csv files. These files were uploaded into a combined Leximancer project for analysis. Leximancer is an online platform which employs a text analytic technique that "processes natural language through a Bayes-inspired algorithmic process to model major thematic and conceptual content from an input text" (Sloan \& QuanHaase, 2017:532). Crofts and Bisman (2010:188) suggest that Leximancer can be used to generate and recognise themes that may be missed if researchers opt to manually code data.

Another benefit of employing the Leximancer software, is that Leximancer not only conducts a conceptual analysis of the data, but also produces a relational analysis (Leximancer, 2017:8; Sloan \& Quan-Haase, 2017:532). In Leximancer, the conceptual analysis measures the presence and frequency of concepts, which may be words or phrases, while the relational analysis identifies how these concepts are "related to each other within the documents (Leximancer, 2017:8). Leximancer therefore determines the cooccurrence of concepts and represents this information visually, showing the most prominent relationships between concepts. Both forms of analysis can be seen in Figure 6 , which shows the initial set of concept seeds and themes generated by Leximancer, before any modifications to the generated list had been made by the researcher.

In this figure, the themes are represented by coloured spheres, which are heat-mapped "to indicate importance" (Leximancer, 2017:12). The most important theme appears in red, followed by the next "hottest" in orange. Cool colours like blue and green denote less important themes (Leximancer, 2017:24). The size of a theme sphere is "has no bearing to its prevalence or importance ... the circles are merely boundaries" (Leximancer, 2013). Dominance of themes is determined by the number of concepts within the theme; a histogram to the right of the concept map shows a 'thematic report', with a synopsis of the most prevalent themes also ranked through colour mapping (Leximancer, 2013).

Concepts are determined by 'seed words', which Leximancer learns from the text itself. These seed words are used to define concepts, "... with each concept definition containing one or more seeds" (Leximancer, 2017:9). The discovery of concepts by Leximancer is dynamic, implying that when a more prevalent term is revealed, the seed words move closer or further away from the centre of the concept's definition. The user can manually provide seed words, or allow Leximancer to identify such words, based on the frequency of appearance of these words within the text (Leximancer, 2017:9). In this study's analysis, the researcher allowed Leximancer to identify concepts initially, before any changes were applied to the concepts. As mentioned, Figure 6 shows the initial theme and concept map generated from the 12 .csv files initially uploaded into the Leximancer project. 
and concepts are shown by dots, which are linked to related concepts. The font size of both the themes and concept labels "denotes its frequency in the text" (Leximancer, 2017:22).

\section{$4 \quad$ App Store Review Findings}

The aim of this analysis was to identify compliments and complaints that app users had regarding existing blood donation apps. Keeping this goal in mind, the researcher explored the Leximancer findings, first from a broad thematic point of view, and then in-depth by each concept. The findings of this analysis, combined with the theoretical framework of the study, informed the questions that were asked in the quantitative online questionnaire, which was administered in the next phase of the study.

\subsection{A Synopsis: Leximancer's Thematic Overview}

Figure 6 shows the Leximancer map, generated after editing the original concept seeds. This figure also illustrates frequent concepts associated with each theme, and the 'Analyst Synopsis' of the overreaching themes, prevalent in the data.

Figure 6: Leximancer thematic map showing concepts and analyst synopsis

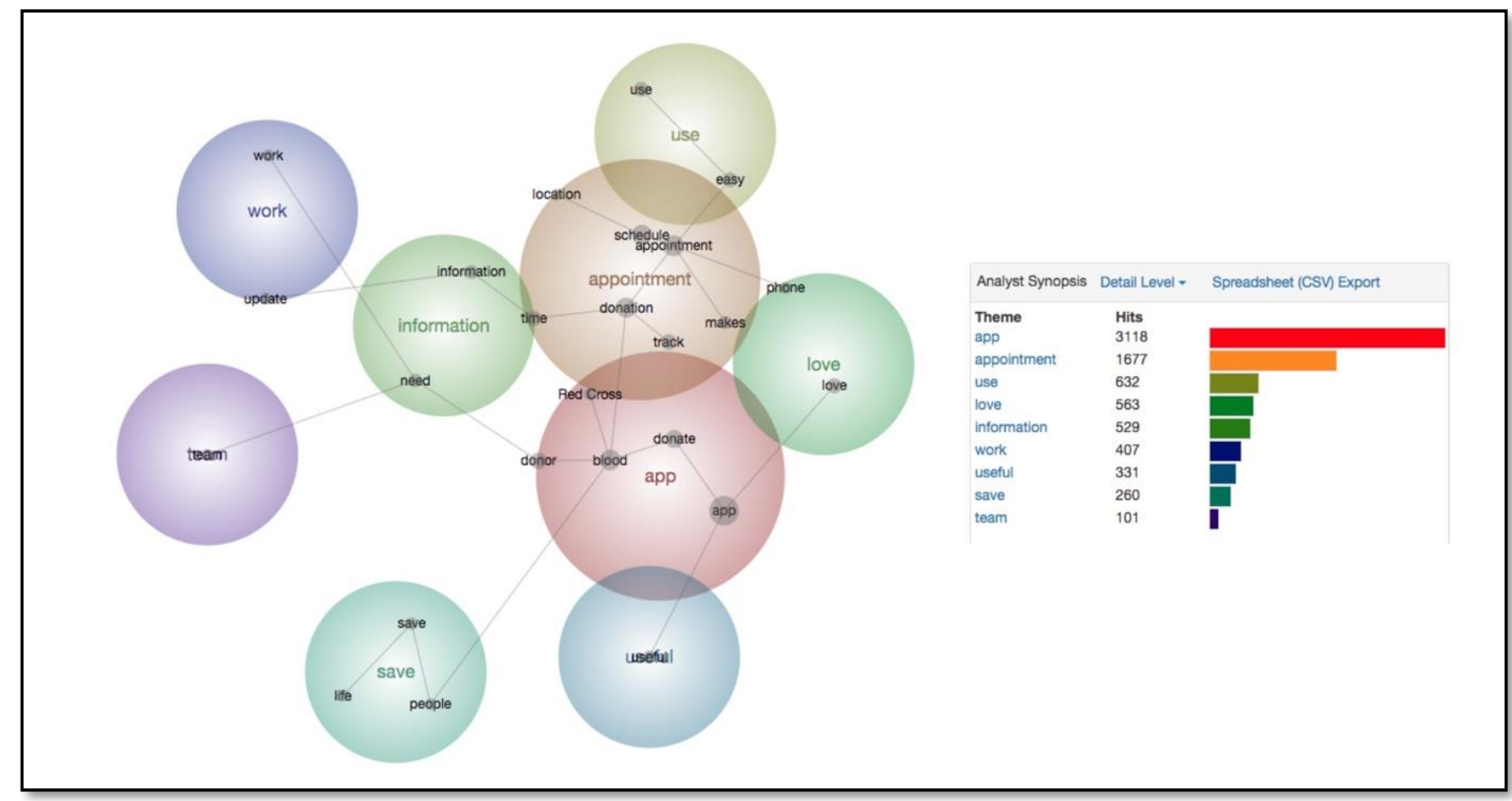

This analysis revealed nine prominent themes within the data. Not surprisingly, 'app' was the most relevant theme, and encompassed the concepts of 'donor', 'blood', 'donate' and 'app'. Considering the research questions of this study, this result is indicative of an emphasis by the data on the focus area of this study: a mobile blood donation app. The next most prominent theme identified within the data, was that of 'appointment'. The concepts within this theme show a strong emphasis by users on scheduling and tracking 
donations through the apps that they had reviewed, as is evident from this excerpt included as evidence for the theme.

"Using this app is so much better than having them call me all the time to schedule, or calling myself. It organi[z]es everything together with my digital donation card and keeps track of my donations."

The theme 'use' focused strongly on the ease of use experienced by the reviewers, as the theme itself included the concepts of 'easy' and 'use'. According to Leximancer (2017:12), "concepts that appear ... attract one another strongly, and so tend to settle near one another in the map space." It therefore stands to reason that the result was also indicative of an ease of use perceived by users when making appointments through the reviewed apps, as the themes of 'use' and 'appointment' showed an overlap beyond just a closeness in proximity on the map.

The next theme ranked in prominence, was that of 'love'. The visible concept in this view, showed that 'love' was strongly linked to 'app', indicating that a notable number of reviewers commented on what their feeling was while using the app they had reviewed. In instances where reviewers felt this typically positive emotion, the reviews seemed to be brief in their conviction, and focused on the theme of 'love'.

"Love it."

"Love this [.....] Such a great job."

"[i]t is [awsome] bro! keep it up i love it."

"Love it."

The theme 'useful' was closely related to the theme 'app', suggesting that the reviewers found the use of the specific app they had reviewed beneficial to their blood donation experience. This correlates with the premise of the Task-Technology-Fit (TTF) theory, where a technology is considered appropriate to facilitate a specific task to the degree to which it assists an individual in performing that task - in this instance, the usefulness identified in the theme relates to the task of donating blood (Irick, 2008).

'Work' was the next most prominent theme identified through Leximancer, and upon further investigation of this theme's concepts, the focus of the reviews associated with this theme seemed to praise the work that had been done by developers of the app, or were commenting needs relating to the functionality of the app. At this stage, the researcher identified the theme 'work' as a focused on the technical aspects of the reviewed apps, which were explored further in the analysis of individual concepts.

The theme 'information' confirmed a strong emphasis on users' need for information, specifically regarding the concept of 'time'. With the slight overlap between 'information' and 'appointment' as themes, specifically with the shared concept of 'time', it became evident that users recognised their need for information to effectively coordinate with the 
relevant blood donation institutions, specifically concerning donation times. Fogg's Behaviour Model (FBM), emphasizes three elements that must converge to catalyse a desired behaviour: motivation, ability and triggers (Fogg, 2009:40). According to FBM, increasing motivation is not always the best course of action, and that "often, increasing ability (making the behaviour simpler) is the path for increasing behaviour performance" (Fogg, 2009:42). Having access to relevant information regarding donation times, could increase donors' ability to follow through with their intention to donate blood.

The theme 'save' showed a prominence regarding the concepts of 'people' and 'life'. This acknowledgement by reviewers of the potential of blood donation apps to save lives, is indicative of altruism, which is typically an intrinsic motivation for action (Legault, 2016). This result is noteworthy, as in the development of mobile apps, especially if the concept of gamification is being considered as a feature, "resting upon intrinsic motivations, whenever possible, is desirable..." (Salomoni, Prandi, Roccetti, Nisi \& Nunes, 2015:131).

The final theme in the analyst synopsis was 'team'. Based on the evident text excerpts supplied by the synopsis, it appeared that users were referring to either backend development or marketing teams, or a feature by the app 'Blood Donor' by the American Red Cross, which allows users to " ... invite others to join them on a lifesaving team" (American Red Cross, 2017).

\subsection{The Essence of a Theme: Leximancer Concepts}

The main goal of this analysis was focused on determining perceptions related to this study's research questions, based on the concepts associated with the main themes identified within the Leximancer analysis. In total, 25 frequent concepts were identified by Leximancer. Figure 7 shows these 25 concepts, in ranked order, from most to least relevant. 
Figure 7: Concepts associated with nine main themes, ranked in order of relevance

\begin{tabular}{|c|c|c|c|}
\hline \multicolumn{3}{|c|}{ Ranked Concepts } & \\
\hline Name-Like & \multicolumn{3}{|c|}{ Count Relevance } \\
\hline Red Cross & $\underline{126}$ & $05 \%$ & $\square$ \\
\hline Word-Like & \multicolumn{3}{|c|}{ Count Relevance } \\
\hline app & $\underline{2376}$ & $100 \%$ & $\square$ \\
\hline blood & $\overline{805}$ & $34 \%$ & \\
\hline appointment & $\overline{713}$ & $30 \%$ & \\
\hline donation & $\overline{701}$ & $30 \%$ & 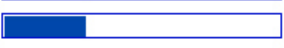 \\
\hline schedule & $\overline{618}$ & $26 \%$ & Г \\
\hline donate & $\overline{593}$ & $25 \%$ & \\
\hline donor & $\overline{507}$ & $21 \%$ & \\
\hline love & $\overline{464}$ & $20 \%$ & \\
\hline use & $\overline{431}$ & $18 \%$ & 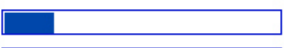 \\
\hline time & $\overline{374}$ & $16 \%$ & \\
\hline useful & $\overline{331}$ & $14 \%$ & \\
\hline easy & $\overline{315}$ & $13 \%$ & \\
\hline track & $\overline{314}$ & $13 \%$ & \\
\hline work & $\overline{287}$ & $12 \%$ & $\square$ \\
\hline information & $\overline{283}$ & $12 \%$ & \\
\hline need & $\overline{268}$ & $11 \%$ & $\square$ \\
\hline makes & $\overline{133}$ & $06 \%$ & $\square$ \\
\hline update & $\overline{122}$ & $05 \%$ & 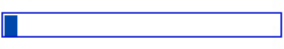 \\
\hline phone & $\underline{115}$ & $05 \%$ & 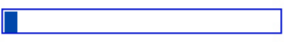 \\
\hline people & $\overline{112}$ & $05 \%$ & $\square$ \\
\hline life & $\overline{110}$ & $05 \%$ & 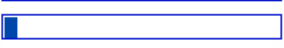 \\
\hline save & $\overline{102}$ & $04 \%$ & 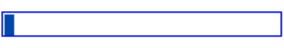 \\
\hline team & $\overline{101}$ & $04 \%$ & 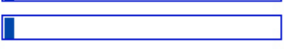 \\
\hline location & $\underline{82}$ & $03 \%$ & $\mathbf{L}$ \\
\hline
\end{tabular}

The researcher focused on this list of concepts during the analysis and relied on the manner and frequency of how concepts related to one another, to further investigate concepts and their meaning. In instances where the researcher was required to search for further clarification of concepts, Leximancer's concept cloud map and thesaurus functions were relied upon. The concept cloud map is also heat-mapped, with hot colours (red, orange) indicating the most relevant concepts, and cool colours (blue, green), representing the least relevant concepts. This map is also interactive, allowing the researcher to click on a concept label " ... to select it and see the list of related concepts in the right-hand tab" (Leximancer, 2017:22). The concept cloud map, list of concepts and the selected concept of 'love', with its related concepts to the right, are shown in Figure 8. 
Figure 8: Leximancer concept cloud map; concept cloud map with concept 'love' and its related terms

\section{Concept cloud map}

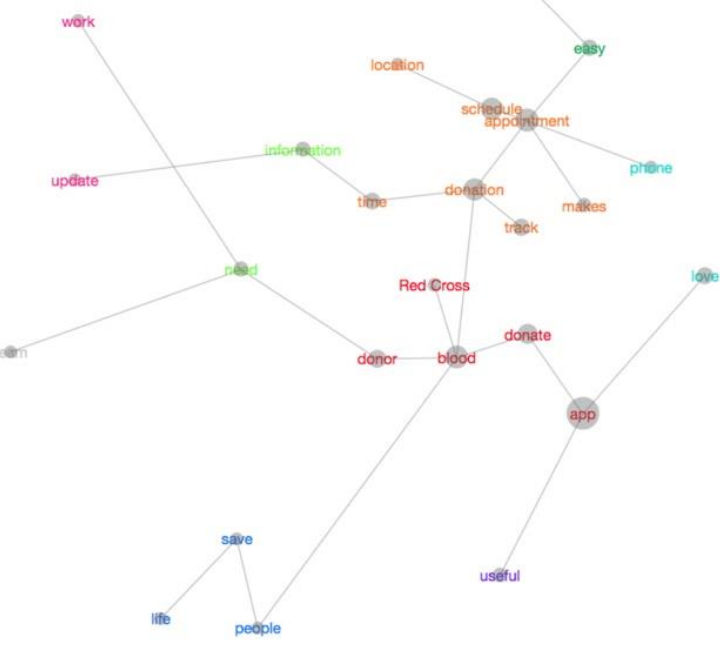

\section{Concept 'love' selected}

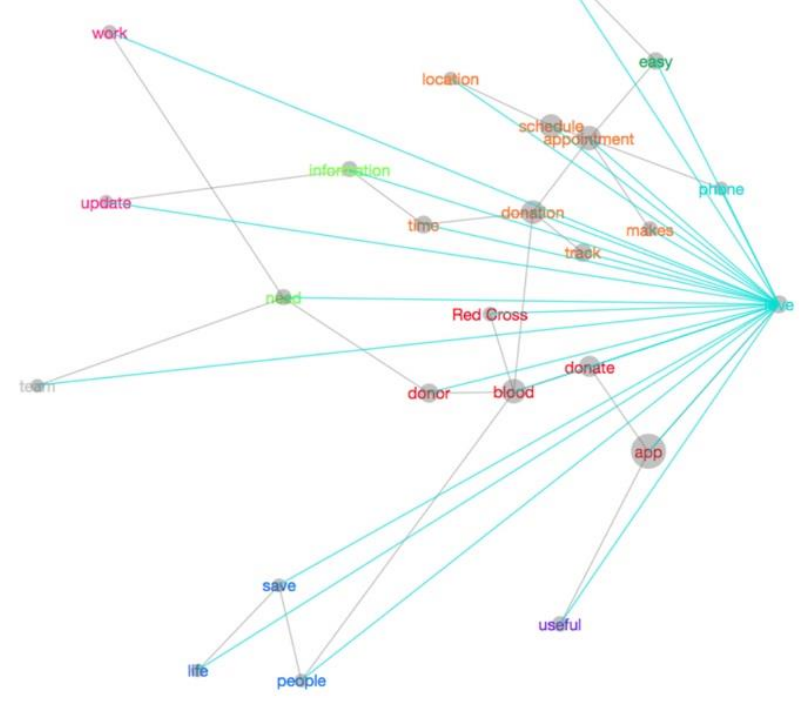

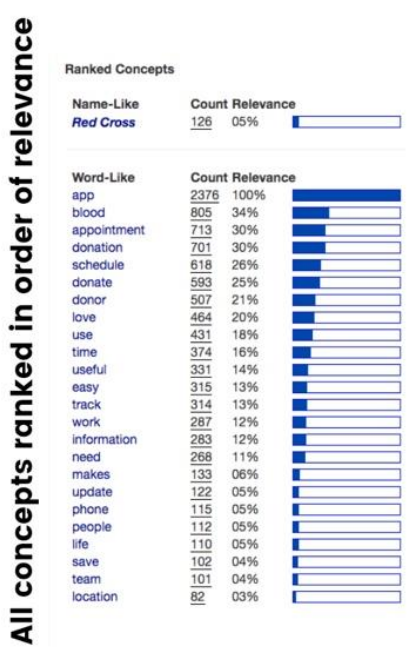

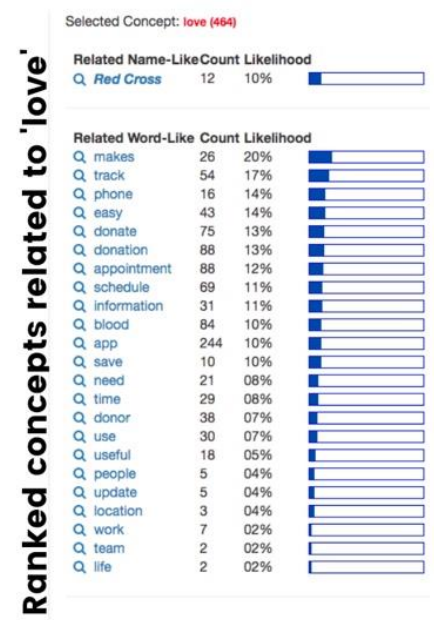

Since a ranked list of the thesaurus words that define and describe each concept is provided by the thesaurus tab in Leximancer, this function was also employed in instances where the terms related to a concept could illuminate the formation, and therefore the meaning, of a concept (Leximancer, 2017:27). 


\subsection{Concept analysis}

Not surprisingly, the 'app' concept was the most relevant of all the concepts. Although the concept of 'makes' was the concept most related to that of 'app', the presentation in the concept cloud view of Leximancer did not aid the researcher in deducing its relevance within the data. Therefore, the thesaurus function within Leximancer was consulted to infer the meaning of 'makes' in relation to 'app'. As mentioned, this strategy was applied throughout the analysis process, to facilitate a better understanding of meaning regarding the relationships between the themes and their associated concepts.

Upon querying the text excerpts provided as evidence for 'makes' within the thesaurus and shown below, it became evident that the prominent use of this concept related to app users perceiving the blood donation app they had reviewed, as an aid in making the process of blood donation 'easy'. The concept 'use' was also shown likely to be combined with the concepts of 'easy', 'schedule' and 'track'. This relationship further confirms the perceptions of users that a mobile blood donation app was enhancing the users' blood donation experience.

"App makes it incredibly easy to schedule time to help out by donating blood!"

"This app is very well put together and makes it incredibly easy to schedule and make blood donations run smoothly. Incredibly user-friendly!"

"This app makes managing my appointments incredibly simple and convenient, and provides a lot of interesting resources about blood donation! Great job!"

"Hard work, thought, and dedication put for this creative app! It makes the donation process extremely easy and convenient for frequent donors."

The concept of 'blood' had a high likelihood to be related to the concepts of 'donate', 'people' and 'makes'. This relation was to be expected, as the focus of the apps was to facilitate people in donating blood, and it had been established that a common perception among reviewers had been that these apps made the process of blood donation easier for users.

The concept of 'track' in relation to 'blood' was explored next, and it was discovered that users found the ability to keep track their blood donation history, and other donor-related information, useful. This assertion was confirmed by those users who complimented an app's ability to facilitate donation tracking, in contrast to those users that complained about certain tracking features not being available, as is evident in the reviews below.

"Great for keeping track!"

"I can never track where my donation went to :("

"Great for keeping track of my giving."

"[Disappointed]! Donated platelets and [can't] track where [it] went." 
The concept 'love' was likely to be used in context with 'makes', 'track', 'easy' and 'phone' illustrating the reviewers' appreciation of the mobile blood donation app making their blood donation more manageable. The concept of 'phone', was most frequently related concept to that of 'donor'. Since the apps that were reviewed were mobile applications, the concept of 'phone' was not surprising. The concept of 'donor' showed a frequent relation to the concepts 'track', 'blood', and 'need'. Since reviewers frequently referred to themselves in the third person as 'donor' in the reviews, it is expected that the concept 'donor' would be related to those terms discussing the process of blood donation, donation tracking and needs identified by the users themselves.

However, worryingly the mention of 'phone' and its related terms was evidently negative and revealed complaints regarding technical issues and operating system compatibility issues, as well as mention of the app being annoying. These reviews highlight a need for a support team assisting users with technical difficulties, or at least an expansive technical support database that users can query online and from within the app.

" ... my dad switched to a Windows phone and now is unable to use the app so it would be nice to have a Windows version."

"Even with good WiFi, it won't let me download from my phone. Please help?"

"Almost as annoying as the weekly phone calls."

"[Wouldn't] even let me make [an] account taking it off my phone."

'Appointment' as a concept was highly likely to be related to the concept of 'schedule'. These two concepts were used interchangeably by users to address the ability to schedule, reschedule, book, and cancel appointments using the apps they had reviewed. In South Africa, blood donors are not able to schedule blood donation appointments with blood donation organisations, as donation opportunities are generally available in a pop-up fashion at malls or companies, that can commit to hosting four viable blood drives annually (South African National Blood Service, 2016).

Although there are numerous donor centres across the country, the public is received in a first-come-first-served manner. Considering the emphasis placed by app users on the ability to schedule appointments, this ability could be an important feature to incorporate in the features of a blood donation app. However, such a feature can only be considered if a systemic change is made by South African blood donation organisations to also allow for the scheduling of appointments.

The likelihood of 'track' as a concept being used in relation to 'donation' was also explored. As mentioned, users placed emphasis of the importance of their ability to track their blood donation history and donor-related information. However, the concept of 'location' was also found to be frequently related to that of 'donation'. Users displayed a need for an app feature that provided them with accurate donation locations, as is evident by reviewers using terms such as 'miles', 'exact', 'south', 'central', 'building', and 'downtown'. Text 
excerpts highlighted the frustration experienced by users when an app's location feature was not accurate, which seemed to be the norm with this feature among the sampled apps.

"Worked well but did not give all location site choices. I had to look up the sponsor site to get location I wanted."

"Need more accurate location. As I am not able to mention exact location after district, city."

"Thumbs up for the initiative. but can't able to access the location kindly fix it."

"Location services are on for this app and it will not even come up on the map. I even searched for the exact address and it will not allow me to select the location that I want."

The concept of 'donate' was also related to the concepts of 'blood', 'makes', 'track', 'location', and 'schedule'. Since a donation is typically the desired outcome of an app user's interaction with a blood donation app, these previously discussed themes related to the concept 'donate' did not reveal any novel findings. However, the concept of 'time' was found to be frequently related to 'donate', and an analysis of the thesaurus terms associated with 'time' revealed that users had addressed the concept of 'time' in broad contexts. Users mentioned 'time' saved, especially through the convenience of a scheduling functionality, and 'time' wasted was addressed by users in terms of technical difficulties, especially those related to installing the reviewed app.

The likelihood that the concept 'useful' would be used with the concepts of 'life', 'save' and 'people', was high. The app reviewers made assertions that a blood donation app could "Save someone's life ... " or that it "Puts the power of saving a life in your hands ...". The relatedness of the concept 'useful' with these assertions, indicated that blood donors may be intrinsically motivated to act in a pro-social manner by donating blood, when they consider that the consequences of their actions could save another's life (Goette, Stutzer \& Frey, 2010:151). As one reviewer noted, "It helps you feel good about yourself, not because you 'need' to, because you 'want' to."

Users also showed a need for evidence of their donations being used, as one reviewer shared disappointment of not being able to track their donation, while another stated that a feature tracking where their donation was used, was a fundamental feature on the app.

"I can never track where my donation went to :("

"I think the coolest part of donating, is seeing where your blood ends up and knowing you may have just helped save someone's life."

'Work' as a concept was most frequently related to the concept of 'need'. The terms and text excerpts associated with these concepts revealed that technical issues, inhibiting the app from working, were mentioned often by reviewers. Reviewers were also vocal about what they needed from the app that they were reviewing, in terms of technical functionality or bug fixes. Some reviews were more helpful than others in explaining the functionality issues and making suggestions. 
"Registration need[s] to be improved."

"Features l'd love to see (in no particular order): ... At a minimum provide a link directly from a scheduled donation in the app to the standard web page; better yet, develop a mobile version of the questions that can be answered from within the app.

The concepts of 'information' and 'update' showed a high likelihood of relatedness. Since 'information' was also related to 'track' and 'time', it became clear that users' comments addressed a need to receive information about donation times and tracking information regarding their blood donation profiles. 'Update' was also related to the concept 'team', which showed contrasting meanings when it was investigated in the thesaurus function. Reviewers commented on 'team' in terms of the functionality of an app to allow users to "join or create a ... team, recruit other blood donors and view rankings on the 'Blood Donor Teams Leaderboard'" (American Red Cross, 2017a). Although the concept of 'team' was ranked low in terms of concept relevance, this finding was significant in the context of gamifying a blood donation experience, as " ... gamification is an alternative for tasks and services that could not attract people before, thanks to the fact of inserting playable elements to the desired service" (Domingos, Lima, Messias, Feijó \& Diniz, 2016:5624).

'Team' was also related to the terms addressing the developmental and marketing teams that are responsible for the creation and maintenance of the apps. In this context, users voiced compliments and raised issues relating to functionality of the apps they had reviewed.

"This is the very good app designed by backend team ... "

"Someone needs to go into the service or backend and figure out where the mobile app connects to and run some regression tests. My previous team doesn't display and when I try to leave the I correctly associated team, it saves me in another team."

By merging this analysis with findings from an initial literature review, the researcher formulated an online interview schedule, that was delivered asynchronously to respondents. The interview questions took every element discovered through the app store review analysis into consideration, ensuring that the concepts that were revealed were presented to the interviewees, and that further insight could be gained into the interviewees' own opinions on these features, concerns and preferences.

\section{Conclusion}

This paper shared the process of sampling and analysing app store reviews for mobile blood donation apps. The purpose of this research was to advise the focus of an asynchronous online interview schedule, which was deployed after the conclusion of the app store review analysis. By employing triangulation in such a manner, the researcher could better understand which questions should be asked in the interview, and could also gain an understanding of typical concerns and compliments that mobile blood donation app users deem important enough to post. Since this paper's research forms part of a larger 
study, its relevance is intensely linked to the goals and outcomes of that study, however the methodology followed here, could prove valuable to other researchers in the field of mobile app design.

\section{References}

AMERICAN RED CROSS. (2017). Red Cross has an app for that. Available from: http://www.redcross.org/news/article/Red-Cross-Has-an-App-for-That

AMERICAN RED CROSS (2017a). Blood app: You hold the power to save lives. Available from: http://www.redcrossblood.org/bloodapp

ANG, S.H. (2014). Research design for business \& management. London: Sage.

ANGELLIST. (n.d). Appbot: Take the pain out of managing app store reviews. Available from: https://angel.co/appbot

ASIF, N. and HASSAN, K. (2015). Voluntary blood donation. Journal of Islamabad Medical \& Dental College (JIMDC), Vol. 5, No. 1, pp. 1-2.

BRYMAN, A. \& BELL, E. (2014). Research methodology: Business and management contexts. Cape Town: Oxford University Press.

BRYMAN, A. \& BELL, E. (2015). Business research methods. International $4^{\text {th }}$ edition. Oxford: Oxford University Press.

CRESWELL, J.W. (2014). Research design. International Student $4^{\text {th }}$ edition. London: Sage.

CROFTS, K. and BISMAN, J. (2010). Interrogating accountability: An illustration of the use of Leximancer software for qualitative data analysis. Qualitative Research in Accounting \& Management, Vol. 7, No. 2, pp.180-207.

DOMINGOS, D.C., LIMA, L.F., MESSIAS, T.F., FEIJÓ, J.V., DINIZ, A.A. and FOGG, B.J. (2009). A behavior model for persuasive design. In Proceedings of the $4^{\text {th }}$ International Conference on Persuasive Technology, p. 40. ACM.

DONG, A., LOVALLO, D. \& MOUNARATH, R. (2015). The effect of abductive reasoning on concept selection decisions. Design Studies, 37:37-58. doi:10.1016/j.destud.2014.12.004

DUNNE, D.D. \& DOUGHERTY, D. (2016). Abductive reasoning: How innovators navigate in the labyrinth of complex product innovation. Organization Studies, Vol. 37, No. 2, pp. 131-159. doi: $10.1177 / 0170840615604501$

EYEWITNESS NEWS. (2016, June 14). SANBS reiterates need for more blood donors. Eyewitness news. Available from: http://ewn.co.za/2016/06/14/SANBS-reiterates-need-for-more-blood-donors

FISCHL, A. (2013). Critical Factors for Successful Mobile Applications. Vienna: Technical University of Vienna. Available from: http://www.semeion.net/qr/thesis mobapp.pdf

FISHER, S. (2016, June 10). SANBS appeals to SA to donate blood this winter. Eyewitness News. Available from: http://ewn.co.za/2016/06/10/SANBS-appeals-to-SA-to-give-blood-this-winter

FOGG, B.J. (2009). A behavior model for persuasive design. In Proceedings of the $4^{\text {th }}$ International Conference on Persuasive Technology, p. 40. ACM. 
GOETTE, L., STUTZER, A. and FREY, B.M. (2010). Prosocial motivation and blood donations: a survey of the empirical literature. Transfusion Medicine and Hemotherapy, Vol. 37, No. 3, pp.149-154.

GRAY, D.E. (2013). Doing research in the real world. $3^{\text {rd }}$ edition. London: Sage.

HENNINK, M., HUTTER, I. \& BAILEY, A. (2011). Qualitative research methods. London: Sage.

HSU, J.S.C., LIN, T.C., FU, T.W. and HUNG, Y.W., 2015. The effect of unexpected features on app users' continuance intention. Electronic Commerce Research and Applications, Vol. 14, No. 6, pp. 418-430.

HUGHES, J. (Editor). (2012). SAGE internet research methods. London: Sage.

IACOB, C. and HARRISON, R. (May 2013). Retrieving and analyzing mobile apps feature requests from online reviews. Proceedings of the 10th IEEE Working Conference on mining software repositories held in San Francisco, CA, USA, pp. 41-44. IEEE.

IRICK, M.L. (2008). Task-technology fit and information systems effectiveness. Journal of Knowledge Management Practice, Vol. 9, No. 3, pp.1-5.

ITBUSINESS. (2014, November 24). Seven must-have features for great mobile apps. Available from: http://www.itbusinessedge.com/slideshows/seven-must-have-features-for-great-mobile-apps-09.html

JOHANNESSON, P. and PERJONS, E., 2014. An introduction to design science. Springer.

JOHN, N. (2016, June, 22). Donate blood, save a life. News24. Available from: http://www.news24.com/SouthAfrica/Local/Maritzburg-Fever/donate-blood-save-a-life-20160621

KHANDAVALLI, K. (2014, May 12). The 5 most popular features in business mobile apps. Available from: http://seventablets.com/blog/the-5-most-popular-features-in-business-mobile-apps/

KUMAR, M. (2015, May 15). Features to make your app popular. Available from: http://www.innofied.com/8key-features-make-app-popular/

LEGAULT, L. (2016). Intrinsic and Extrinsic Motivation. In Encyclopedia of Personality and Individual Differences, pp. 1-4. Springer International Publishing.

LEXIMANCER. (2013). FAQ: Why isn't the biggest theme circle on the concept map the top one in the associate thematic summary report tab? Available from: https://info.leximancer.com/faq-displays-andoutputs/

LEXIMANCER. (2017). Leximancer user guide release 4.5. Available from: http://doc.leximancer.com/doc/LeximancerManual.pdf

MASSUNG, E., COYLE, D., CATER, K.F., JAY, M. and PREIST, C. (April 2013). Using crowdsourcing to support pro-environmental community activism. In Proceedings of the SIGCHI Conference on Human Factors in Computing Systems, pp. 371-380. ACM.

MOBILE MARKETING ASSOCIATION SOUTH AFRICA. (2015). MMA SA mobile report: AMPS2015A. Available from: http://cdn1.digicelmore.mobi/images/88/content/MMA-SA-Mobile-Report-AMPS2015A-LR.pdf

OUHBI, S., FERNÁNDEZ-ALEMÁN, J.L., POZO, J.R., EL BAJTA, M., TOVAL, A. \& IDRI, A. (2015). Compliance of blood donation apps with mobile OS usability guidelines. Journal of Medical Systems, Vol. 39, No. 6, pp. 1-21. 
OUHBI, S., FERNÁNDEZ-ALEMÁN, J.L., TOVAL, A., IDRI, A. \& POZO, J.R. (2015). Free blood donation mobile applications. Journal of Medical Systems, Vol. 39, No. 5, pp. 1-20. doi: 10.1007/s10916-0150228-0

PICKARD, A.J. (2012). Research methods in information. London: Facet publishing.

POWERREVIEWS. (2014). The power of reviews: How ratings and reviews influence the buying behavior of the modern consumer. Available from: https://www.powerreviews.com/wpcontent/uploads/2016/04/PowerofReviews 2016.pdf

PULE, P.I., RACHABA, B., MAGAFU, M.G.M.D. \& HABTE, D. (2014). Factors associated with intention to donate blood: sociodemographic and past experience variables. Journal of Blood Transfusion. doi: $10.1155 / 2014 / 571678$

REACT TECHNOLOGY. (2016). Calendar for good, version 0.1, mobile app, viewed 11 July 2016 <https://play.google.com/store/apps/details?id=technology.react.calendarforgood\&hl=en>

RUIZ, I.M., NAGAPPAN, M., ADAMS, B., BERGER, T., DIENST, S. and HASSAN, A. (2017). An examination of the current rating system used in mobile app stores. IEEE Software. doi: https://doi.org/10.1109/MS.2017.265094809

RUSSEL, B. (2014). Stanford Encyclopedia of Philosophy: A priori justification and knowledge. Available from: https://plato.stanford.edu/entries/apriori/\#PriJusResSomNonSouEvilntRatIns

SALMONS, J. (2016). Doing qualitative research online. London: Sage.

SALMONS, J. (2015). Qualitative online interviews. $2^{\text {nd }}$ edition. London: Sage.

SALOMONI, P., PRANDI, C., ROCCETTI, M., NISI, V. \& NUNES, N.J. (2015). Crowdsourcing urban accessibility: Some preliminary experiences with results. In Proceedings of the $11^{\text {th }}$ Biannual Conference on Italian SIGCHI Chapter, pp. 130-133. ACM.

SAUNDERS, M., LEWIS, P. \& THORNHILL, A. (2012). Research methods for business students. $6^{\text {th }}$ edition. Harlow: Pearson Education.

SEFFERMAN, A. (2016). The average mobile apps' ratings and reviews, by category. Available from: https://www.apptentive.com/blog/2016/10/20/average-mobile-apps-ratings-and-reviews-by-category/

SLOAN, L. and QUAN-HAASE, A. (Editors). (2017). The SAGE handbook of social media research methods. Sage.

SOUTH AFRICAN NATIONAL BLOOD SERVICE. (2016). Blood drives: Host a blood drive. Available from: https://sanbs.org.za/blood-drives/

STATISTA. (2016). Number of aps available in leading app stores as of June 2016. Available from: http://www.statista.com/statistics/276623/number-of-apps-available-in-leading-app-stores/

WOOLDRIDGE, D. and SCHNEIDER, M. (Editors). (2010). The business of iPhone app development: Making and marketing apps that succeed. Apress. 